\title{
VÍCTIMAS DE ABUSO SEXUAL ESPECIALMENTE VULNERABLES: OBTENCIÓN DEL TESTIMONIO
}

\section{Especially vulnerable victims of sexual abuse: obtaining testimony}

\author{
Alba Vara* \\ Universidad Complutense de Madrid
}

Antonio L. Manzanero**

Universidad Complutense de Madrid

Rocío Vallet $^{* * *}$

Universidad Pontificia de Comillas

* Doctora en Psicología. Psicóloga forense e investigadora del grupo de investigación UCM en Psicología del Testimonio (España). ORCID iD: 0000-0003-1960-7737. Contacto: alba_vara@ hotmail.es

** Doctor en Psicología. Profesor de la Universidad Complutense de Madrid (España). Director del grupo de investigación UCM en Psicología del Testimonio. ORCID iD: 0000-0003-2619-4571. Contacto: antonio.manzanero@psi.ucm.es

*** Doctora en Psicología. Profesora de la Universidad Pontificia de Comillas (España). Psicóloga Forense e investigadora del grupo de investigación UCM en Psicología del Testimonio. ORCID iD: 0000-0003-2674-2435. Contacto: rvallet@cop.es 


\section{Resumen:}

En los delitos sexuales, las características especiales de algunas personas las convierten en víctimas potencialmente vulnerables. Ante la responsabilidad y obligatoriedad legislativa de actuar de manera inmediata en defensa de sus derechos y garantizar su protección, los profesionales del contexto policial y judicial que intervienen en la obtención del testimonio tienen el reto de adaptar los procedimientos para la obtención y evaluación de sus testimonios. Las investigaciones previas confirman desinformación y consiguiente mala praxis policial y judicial en los casos de abuso sexual a víctimas especialmente vulnerables: alta demora en las denuncias, repetición de interrogatorios y uso de preguntas inadecuadas. En el presente artículo se analizan todos estos factores de influencia y se dan algunas recomendaciones para mejorar los procedimientos de obtención de sus testimonios. Finalmente, se alerta sobre la posible sugestión que podría derivarse del mal uso de procedimientos que pretenden mejorar la cantidad de información recuperada, pero que pueden contribuir a generar falsas memorias.

\section{Abstract:}

In sexual crimes, the special characteristics of some people make them potentially vulnerable victims. Faced with the responsibility and legislative obligation to act immediately in defense of their rights and guarantee their protection, the professionals of the police and judicial context who intervene in obtaining the testimony have the challenge to adapt the procedures for obtaining and evaluating their testimonies. Previous investigations confirm misinformation and consequent police and judicial malpractice in cases of sexual abuse of especially vulnerable victims: high delay in complaints, repeated interrogations and use of inappropriate questions. This article analyzes all these influencing factors and gives some recommendations to improve the procedures for obtaining their testimonies. Finally, it warns about the possible suggestion that could derive from the misuse of procedures that seek to improve the amount of information recovered, but that can contribute to generating false memories.

\section{Palabras clave:}

Testimonio - Víctima - Abuso sexual - Menores de edad - Discapacidad intelectual 


\section{Keywords:}

Testimony - Victim - Sexual abuse - Minors - Intellectual disability

\section{Sumario:}

1. Introducción - 2. Entrevista a personas especialmente vulnerables - 3 . Entrevista a menores de edad -4. Entrevista a personas con discapacidad intelectual -5 . Testimonio como prueba preconstituida -6 . Ayudas al recuerdo -7 . Falsas ayudas al recuerdo -8 . Conclusiones -9 . Lista de referencias

\section{INTRODUCCIÓN}

El procedimiento empleado en la obtención del testimonio cobra particular interés en aquellos delitos en los cuales la declaración del testigo es la única prueba para demostrar la comisión de los hechos. Esto sucede especialmente en los casos de delitos contra la libertad e indemnidad sexual, particularmente, cuando implican a víctimas menores de edad y a personas con discapacidad intelectual (DI, en adelante), dos colectivos con necesidades especiales de protección que requieren individual atención.

En multitud de ocasiones, esta tipología delictiva apenas brinda evidencias físicas en las que basar el ulterior juicio de culpabilidad y, por ende, las autoridades judiciales se han de valer exclusivamente en el relato de los hechos vividos por las víctimas. Si, en suma, dicha vulneración de libertad e indemnidad sexual ha sido practicada sobre una víctima especialmente vulnerable, la obtención del recuerdo autobiográfico y la posterior valoración de la credibilidad de este resultará una tarea de gran complejidad.

Para definir a una víctima como especialmente vulnerable la jurisprudencia valora dos tipos de criterios: i) las características propias de la víctima y ii) el tipo de delito cometido. Bajo el primer criterio se engloban a aquellas víctimas que corren el riesgo de sufrir una victimización secundaria y cuyos testimonios pueden verse gravemente afectados por procedimientos inadecuados de investigación; es decir, menores de edad, personas con DI, personas con trastornos mentales y ancianos de avanzada edad. Por otra parte, en función del tipo de delito se consideran especialmente vulnerables las víctimas de delitos sexuales, violencia de género y trata de personas.

A este respecto, la mayoría de los trabajos se han centrado en las víctimas de abuso sexual (AS) menores de edad y personas con DI. La bibliografía al res- 
pecto señala la elevada probabilidad en ambos colectivos de llegar a ser víctimas de delitos sexuales (Márquez et al., 2017), aunque la prevalencia y dimensión real de los delitos sexuales en estas víctimas se desconoce a consecuencia de las limitadas tasas de denuncia. Este hecho, unido a la presunción de su incapacidad para aportar testimonio válido, acrecienta aún más su vulnerabilidad.

En particular, los menores de edad componen un colectivo de víctimas especialmente vulnerable para este tipo de delitos, debido a las limitaciones sobrevenidas por su desarrollo evolutivo y a la carencia de conocimientos y experiencia acerca de la sexualidad (Manzanero y Barón, 2014). De igual modo, las personas con DI son consideradas víctimas vulnerables por su elevada dependencia y sumisión a terceras personas, así como por la falta de intimidad y educación sexual (Verdugo et al., 2002) recibida. En ocasiones, estas últimas presentan conjuntamente un déficit de habilidades sociales y/o comunicativas que les impide detener las agresiones o denunciarlas a posteriori. Adicionalmente a estas limitaciones propias del desarrollo o sobrevenidas por la DI, la vulnerabilidad de ambos colectivos se ve acrecentada por la falta de procedimientos para la obtención y valoración de su declaración, el uso inadecuado de los existentes y por la reducida credibilidad atribuida a su testimonio (Manzanero, 2000), especialmente en los casos de víctimas menores de edad (Cederborg y Hellner, 2010) y mujeres con DI (Martorell y Alemany, 2017).

Sin embargo, lejos de la creencia común, las personas con DI pueden tener habilidades y aptitudes para realizar muchas otras tareas (Seoane, 2004), equiparando su capacidad para testificar a la de la población normal cuando se realizan las adaptaciones necesarias (Manzanero et al., 2012). No obstante, la mayoría de estos casos requieren apoyos especiales (Milne y Bull, 2006), por ejemplo, mediante un facilitador (Recio et al., 2012). La figura del facilitador surgió como un profesional de la psicología independiente y neutral, experto en DI y en la evaluación de las capacidades cognitivas que afectan al proceso judicial, que asiste a la persona con DI en su comunicación durante el proceso policial y judicial y ofrece los apoyos necesarios para garantizar un testimonio válido y fiable (Recio et al., 2012). Actualmente se ha ampliado para dar apoyo a personas investigadas con DI, donde se facilitará - además - la evaluación de su capacidad procesal, incluyendo el derecho a una asistencia experta en aquellas víctimas especialmente vulnerables que se vean limitadas o imposibilitadas para valerse por sí mismas en el ejercicio de sus derechos o en el cumplimiento de sus obligaciones procesales; y, por consiguiente, requieran un procedimiento adaptado a su particular situación. 
De igual forma, los menores de edad tienen una competencia notable para informar de un recuerdo autobiográfico; sin embargo, la cantidad y la calidad de la información que aportan puede verse condicionada por las estrategias empleadas en la obtención de sus declaraciones (Lamb y Brown, 2006). Así, en la práctica, es frecuente encontrar actuaciones de profesionales en las que predomina la obtención del testimonio mediante preguntas cortas, muchas veces sugestivas, más que como relato libre de los hechos, pudiendo contribuir a la alteración de la huella de memoria original (Chrobak et al., 2015).

Por consiguiente, debido a las limitaciones de los métodos tradicionales de obtención y valoración de la credibilidad del testimonio en víctimas especialmente vulnerables, algunos autores señalan la importancia de emplear protocolos de entrevista, válidos y fiables, adaptados a las características especiales de la víctima, como - por ejemplo- el protocolo NICHD $^{1}$ (Lamb et al., 2007).

\subsection{Víctimas con necesidades especiales de protección}

En el caso de las víctimas infantiles, la relevancia de los procesos de toma de declaración se ha visto reflejada progresivamente en la jurisprudencia, considerándose prioritario preservar su intimidad y su desarrollo evolutivo ante las comparecencias judiciales, adecuando el procedimiento a las necesidades especiales, habilidades, edad, madurez intelectual, capacidad y lenguaje del menor; evitando así múltiples intervenciones².

El interés superior de los menores de edad debe actuar a modo de guía en cualquier medida o decisión que haya de tomarse con relación a los mismos. En España, los derechos de los menores de edad y las personas con discapacidad víctimas de delitos, quedan recogidos en el Estatuto de la Víctima del Delito ${ }^{3}$, a partir de la Convención sobre los Derechos de las Personas con Discapacidad y de la Convención de los Derechos de los Niños y las Niñas de las Naciones Unidas. Como principios básicos se establecen: el respeto a la dignidad, la autonomía individual, la no discriminación y la accesibilidad a la justicia; debiéndose adoptar las medidas oportunas para ello.

En materia de exploración de menores de edad con desarrollo típico y personas con DI, el artículo 23 de la Ley $N^{\circ}$ 4/2015 clasifica a ambos colectivos

Para una traducción y adaptación al español puede verse González y Manzanero (2018).

Apartados № 14 y 23 de la Resolución №2005/20 del Consejo Económico y Social de la Organización de las Naciones Unidas, ECOSOC.

3 Ley $\mathrm{N}^{\circ} 4 / 2015$ 
como "víctimas con necesidades especiales de protección", dadas las características personales y evolutivas que les hacen especialmente vulnerables a la victimización secundaria por su participación en el proceso policial y penal. Este reconocimiento da lugar a una serie derechos contemplados en la Ley que velarán por que:

- No se distingan franjas de edad, aplicando las disposiciones legales a todo menor de 18 años y atendiendo a la edad que posean en el momento de prestar declaración.

- Tengan un tratamiento específico, orientado a su protección y asistencia, así como a la calidad objetiva del testimonio que han de prestar.

- En las causas relativas a abusos y agresiones sexuales, se extremen las precauciones y la calidad de la investigación, otorgándoles mayor prioridad respecto a otras pesquisas criminales y agilizando el proceso penal, especialmente cuando se trate de víctimas.

- Se eviten re-exposiciones a múltiples exploraciones (médicas, psicológicas, etc.), debiendo realizar evaluaciones conjuntas en la medida de lo posible y dando pautas de actuación en la toma de declaración para pre-constituir la prueba.

- Cuando los peritos desaconsejen la declaración en juicio oral, se considere el informe pericial psicológico y la grabación de la exploración como pruebas preconstituidas ${ }^{4}$, especialmente en víctimas de delitos contra la libertad e indemnidad sexual menores de 14 años.

- Se puedan adoptar otras medidas de cautela como la videoconferencia, utilización de mamparas, espejos unidireccionales u otros medios análogos para evitar los efectos de la re-victimización.

- Se modifique el lenguaje, empleando un lenguaje claro, sencillo y accesible, adoptando una terminología adecuada al nivel de desarrollo y facilitando la asistencia de apoyos para que la víctima o testigo pueda hacerse entender ${ }^{5}$.

- Se inicie la exploración con recuerdo libre y se continúe con preguntas abiertas cada vez más estructuradas con la finalidad de obtener detalles adicionales, evitando preguntas sugestivas o coactivas.

$4 \quad$ Artículo 443 y 448 de la Ley de Enjuiciamiento Criminal.

5 Artículo 4 de la Ley $N^{\circ} 4 / 2015$ 
- Se realice la exploración en dependencias especialmente concebidas para ello o adaptadas a tal fin, llevada a cabo por profesionales que hayan recibido una formación especial, preferiblemente por una misma persona y, del mismo sexo que la víctima cuando esta así lo solicite ${ }^{6}$.

\section{ENTREVISTA A PERSONAS ESPECIALMENTE VULNERABLES}

En la exploración de las víctimas especialmente vulnerables practicada con el interés de que aporten un testimonio acerca de los hechos delictivos vividos, el profesional encargado de la realización de la entrevista debe evitar dar por sentado que conocen el significado de los términos que emplean (p. ej., con el concepto "relación sexual" pueden estar hablando solo de besos), asimismo, deberá analizar la competencia de la víctima para prestar declaración y sus conocimientos sobre la materia (Manzanero et al., 2013).

De igual modo, el profesional a cargo de la entrevista debe ser especialmente cauto con la formulación de preguntas tendenciosas y sugestivas, por ejemplo, sobre el consentimiento o el grado de consenso en la práctica sexual. Las investigaciones al respecto señalan la importancia de cuidar la formulación de las preguntas y la evaluación de las respuestas, especialmente, cuando estas impliquen la culpabilidad de la propia víctima en actos de carácter sexual, demostrando lo comunes que son dichas implicaciones en la práctica policial hacia estos colectivos y las consecuencias que puede derivar de un mal ejercicio (Antaki et al., 2015.)

\subsection{Factores de influencia en la obtención de la declaración}

En los procedimientos de toma de declaración, tanto en la investigación policial como en la judicial, el objetivo prioritario es profundizar en el testimonio en busca de un relato lo más completo y preciso posible, protegiendo siempre los derechos del testigo. Se tratará de favorecer la recuperación de la información autobiográfica almacenada en la memoria de la víctima.

Esta fase de recuperación ofrece al entrevistador la oportunidad de influir sobre los procesos de la meta memoria, de manera tanto positiva como negativa, y en la exactitud del recuerdo, por lo que se debe prestar especial atención a las variables que afectan a este proceso.

En consecuencia, las demandas cognitivas, la naturaleza desafiante y estresante de tales sucesos, la pericia del entrevistador y el grado de colaboración

$6 \quad$ Artículo 25 de la Ley Nº 4/2015. 
del entrevistado definirán el rendimiento de esta fase, pero, además, existen una serie de variables, descritas a continuación, que intervienen en el proceso de toma de declaración y de cuyo control derivan mejoras en el rendimiento de la tarea de recuerdo.

\subsubsection{Variables asociadas al procedimiento de entrevista}

\section{Formato de recuperación: narrativo vs interrogativo:}

Comúnmente, son escasas las ocasiones en las que el testigo o víctima aporta una descripción completa y exacta de los hechos. Existen dos tipos de acceso y recuperación de los recuerdos: incidental (automática, mediante activación indirecta de los esquemas adecuados) o intencional (elaborada, mediante procesos conscientes y controlados de búsqueda de huellas), los cuales, a su vez, guardan relación con los dos formatos de recuperación empleados en los procesos de toma de declaración: narrativo (por recuerdo libre) e interrogativo (por formulación de preguntas).

En el primer caso, la cantidad de información obtenida es menor, pero más precisa, mientras que, en el formato interrogativo, al dirigir el recuerdo mediante preguntas, la susceptibilidad a influencias externas es mayor $y$, por tanto, aumenta la probabilidad de comisión de errores y distorsión del recuerdo por efecto de información post-suceso (Lane et al., 2001). Es decir, con el formato narrativo se obtiene información más exacta y con el formato interrogativo la información obtenida es más completa, de hecho, al menos la mitad de la información declarada se recuerda a demanda de las preguntas abiertas (Pipe et al., 2004). El recuerdo mediante preguntas mejora cuando no se fuerza a la víctima a emitir una respuesta sobre la cual tiene dudas (Chrobak et al., 2015).

Sin embargo, tal y como recogen algunas investigaciones, en la práctica profesional, habitualmente, los investigadores no se adhieren a los principios de buenas prácticas (Brown y Lamb, 2015), generando inconsistencias como resultado de la formulación de preguntas sugestivas (Andrews y Lamb, 2014). Así pues, la redacción sesgada de las preguntas influye particularmente en la exploración de menores de edad en materia de sugestibilidad y exactitud de la memoria, siendo particularmente perjudiciales las preguntas que sugieren la respuesta (Stern, 1906, citado en Whipple, 1909).

Pese a ello, el procedimiento tradicionalmente utilizado en la toma de declaración supone formular excesivas preguntas cerradas y sugerentes, de respuesta corta y con una secuencia inapropiada, disminuyendo la tasa de concentración del interrogado y desaprovechando el recuerdo de toda aquella 
información no preguntada, al no dar continuidad a los comentarios realizados (Baugerud et al., 2020).

\subsubsection{Variables asociadas al entrevistador/a}

\section{i. Formación y capacitación del entrevistador:}

Es relevante que la persona encargada de la exploración de la víctima o testigo sea un profesional capacitado y experto en memoria y testimonio. El nivel de especialización requerido a estos profesionales deberá ser elevado, disponiendo de habilidades sociales y de comunicación fundamentales ( $p$. ej., la escucha activa), de técnicas generales y específicas de manejo de la entrevista policial y de procedimientos expertos oportunos de intervención en casos especialmente complejos (González y Manzanero, 2018). De igual modo, beneficiará el grado de flexibilidad en el empleo de la técnica que permita la adaptación a las circunstancias personales, materiales y temporales.

Además, repercute positivamente en la precisión del recuerdo, la construcción de una buena relación entre víctima y experto, disminuyendo los efectos sugestivos de la información errónea ante entrevistadores solidarios que brindan apoyo (Saywitz et al., 2019). Recientemente, se ha comprobado que una intervención basada en apoyos y vínculos profesional-menor aumenta en torno al $10 \%$ la disposición del menor a realizar revelaciones y que estas sean creíbles (Hershkowitz y Lamb, 2020), la confianza y disposición a declarar y la cantidad de información aportada (Blasbalg et al., 2019). Si bien un estudio sobre AS en víctimas de 5 a 15 años (Teoh y Lamb, 2013) sugiere que, así como el apoyo facilita la apertura del menor y narración de los hechos, una excesiva locuacidad del entrevistador mengua la disposición de informar en los niños. En definitiva, adoptar una postura empática, aunque respetando el espacio, la objetividad e imparcialidad, reduce los sesgos.

Por otro lado, ante la realización de entrevistas repetidas, valerse del mismo entrevistador mejora el rendimiento y la consistencia del recuerdo (Bjorklund et al., 2000). Se ha comprobado que la presencia de otras personas en la entrevista (familiares, abogados, educadores, etc.) puede sugestionar a la víctima y perjudicar su concentración, por ende, es recomendable que no estén presentes, o al menos dentro de su campo visual, personas de apoyo social o familiares. No obstante, el entrevistador podrá disponer de la presencia de otro profesional igualmente capacitado como ayudante, para la grabación y transcripción del relato, y para la anotación de aquellos aspectos dudosos a especificar previo a la conclusión de la entrevista. 


\section{ii. Comunicación:}

El estilo de comunicación empleado por el entrevistador influye sobre la capacidad y la voluntad de participar activamente en la conversación de víctimas especialmente vulnerables (Lamb y Brown, 2006). El entrevistador debe facilitar la comunicación evitando un estilo dirigido, valiéndose de habilidades básicas de comunicación como la escucha activa, la empatía o la capacidad de resumir y realizar preguntas compatibles con el desarrollo evolutivo del entrevistado.

En aquellos casos en los cuales las limitaciones evolutivas o del desarrollo intelectual condicionen la comunicación, dificultando la aportación del testimonio - aun cuando la memoria es óptima - se solicitará asesoramiento de personal especializado. El nivel de desarrollo lingüístico y cognitivo de la víctima debe marcar las exigencias comunicativas de la exploración, las cuales no deben superar su nivel de comprensión y memoria con el lenguaje empleado en la formulación de las preguntas y teniendo en cuenta las limitaciones en determinadas habilidades o experiencias de aprendizaje (p. ej., problemas de estimación de frecuencias y tiempos de ocurrencia de los hechos) (Bull, 2010). Así, la calidad de la declaración de víctimas con dificultades de comunicación dependerá de la complejidad del lenguaje empleado por el profesional y de la capacidad de la víctima para usar el lenguaje y resistirse a preguntas sugestivas, pudiendo mejorarse mediante instrucciones precisas y preparación (Canning y Peterson, 2020).

De igual modo, se ha observado que el lenguaje no verbal percibido también afecta a la memoria, propiciando la implantación de información (Gurney et al., 2013). Almerigogna, Ost, Akehurst y Fluck (2008) concluyeron que los niños explorados por un entrevistador con un estilo de comportamiento gestual no solidario producen recuerdos más inexactos.

\subsubsection{Variables asociadas al testigo}

\section{i. Desarrollo evolutivo:}

El desarrollo evolutivo es un proceso complejo, continuo e irreversible de cambios madurativos a través del cual se adquieren una serie de habilidades (cognitivas, lingüísticas, socioemocionales y morales). Caso, Arch, Jarne y Molina (2011) destacan la limitada orientación espaciotemporal y la deseabilidad social mostrada hacia los adultos entre los menores de edad preescolar (de 3 a 6 años); la mayor capacidad de atención y memoria, y menor capacidad de sugestión entre los menores de edad escolar (de 6 a 9 años); la capacidad empática, de comprensión y comunicación que presentan durante la pubertad 
(de 9 a 12 años); y el desarrollo de todas éstas capacidades parejas a las adultas en la adolescencia (de 12 a 18 años).

Sin embargo, debemos tener en cuenta que la edad madurativa no tiene por qué coincidir con la cronológica, lo que puede llevar a conclusiones erróneas. Por tanto, resulta de especial interés conocer el desarrollo evolutivo de la víctima a la hora de su exploración.

\section{ii. Capacidad para testificar:}

Las limitaciones sobrevenidas del desarrollo madurativo o del grado de DI de la víctima han de ser tenidas en cuenta en la evaluación específica de su competencia para testificar, previo a la valoración de la exactitud del testimonio, especialmente ante casos de víctimas con DI (Contreras et al., 2015, Silva et al., 2018.). Es importante conocer previamente si el sujeto necesita una adaptación de los procedimientos de obtención de la prueba testifical en función de su capacidad para aportar un relato sobre los hechos.

En particular, en personas con desarrollo típico no es hasta los 3-5 años cuando se desarrolla la teoría de la mente, y, a partir de los 6 años, la capacidad de memoria es cualitativamente similar a la de los adultos.

En el caso de víctimas con DI, las limitaciones afectan principalmente a dos áreas: el funcionamiento intelectual y la conducta adaptativa. Ante la sospecha de una posible DI, se recomienda evaluar su capacidad para testificar para poder realizar adaptaciones si fuera necesario (Alemany et al., 2012). El principal problema radica en la heterogeneidad de este colectivo (por las diversas etiologías y esferas de afectación) y en la consiguiente dificultad de establecer los tipos de déficits asociados a los diferentes grados de DI (Manzanero et al., 2013). Por tanto, será primordial la evaluación de las necesidades y características de la víctima con el objetivo de optimizar y facilitar la entrevista y el posterior análisis de la información, mediante instrumentos creados para tal fin como el CAPALIST (Contreras et al., 2015, Silva et al., 2018).

\section{ENTREVISTA A MENORES DE EDAD}

Los niños son igual de capaces que los adultos a la hora de proporcionar información precisa sobre sus experiencias (Luus y Wells, 1992); no obstante, esta capacidad se ve mermada, no solo por las cualidades de sus recuerdos, sino también por factores cognitivos y sociales, como la inteligencia, la capacidad de lenguaje, los conocimientos previos, la deseabilidad social y la autoridad del entrevistador (Pipe et al., 2004). 
Pese a existir variabilidad interindividual, la capacidad para evocar y describir unos hechos está considerablemente dominada por la variable edad. No es hasta los 3-5 años cuando se desarrolla la memoria episódica, estando en consecuencia más capacitados para aportar un recuerdo exacto y sensible a la sugestión e imaginación cuanto más mayores sean, gracias al aumento de la capacidad para diferenciar el esquema general de los detalles periféricos, de la cantidad de conocimientos oportunos para reconstruir el pasado y de la capacidad de recordar una mayor cantidad de detalles (Bruck et al., 1995, Peterson y Bell, 1996).

Por lo tanto, se refleja una mayor consistencia en el recuerdo (Peterson et al., 2011) y cantidad de detalles en los relatos infantiles a medida que aumenta la edad (Lamb et al., 2003). Peterson, Hallett y Compton-Gillingham (2018), en un estudio prospectivo sobre la capacidad de recuerdo a lo largo de ocho años, hallaron que el recuerdo consistente de la información original es el doble en los niños mayores de 6 años (una media de un 28\%) que en los niños de 4 y 5 años (menos de un 10\%).

Sin embargo, no solo las capacidades cognitivas de la víctima en el momento de la recuperación influyen en la exactitud de las declaraciones, análogamente, las capacidades de codificación con las que contase cuando sucedieron los hechos influyen sobre el recuerdo original (Vallet et al., 2017), disminuyendo así la precisión de la memoria para aquellos delitos cometidos contra víctimas con escaso desarrollo madurativo.

\subsection{Factores a considerar}

\section{i. Sugestibilidad:}

La sugestibilidad es una construcción compleja y multidimensional en la cual intervienen tanto fuentes internas como externas. McFarlane, Powell y Dudgeon (2010) predijeron que la inteligencia, el estado socioeconómico, la capacidad de memoria y el género son factores que contribuyen a la sugestibilidad. De modo que, en niñas con menor coeficiente intelectual, un bajo estado socioeconómico y rendimiento cognitivo, incrementa la probabilidad de sugestión.

El grado de desarrollo evolutivo también actúa como fuente de influencia interna en la subjetividad; sin embargo, su influjo se ve mermado cuando entran en juego otras fuentes externas como los métodos de entrevista de alta presión (Finnilä et al., 2003). A su vez, el recuerdo demorado y los errores en el control de la fuente contribuyen a la sugestibilidad (Volpini et al., 2016). 


\section{ii. Ansiedad y miedo:}

El estrés generado por la situación de toma de declaración merma las capacidades de la víctima y la calidad del relato, al mismo tiempo que aumenta la probabilidad de victimización secundaria.

Es habitual encontrar en los menores de edad preocupaciones, inseguridades y miedos fomentados por una concepción errónea sobre su rol, sobre el proceso y los profesionales encargados de la exploración. Por ejemplo, temen ser castigados si se equivocan, el enfado de la familia por las consecuencias derivadas o la venganza del acusado (Foster, 2017).

Especialmente vulnerables a padecer ansiedad y miedos son los testigos de menor edad ante tareas de recuerdo de hechos desagradables, como situaciones de AS (Foster, 2017). Las preocupaciones, inseguridades y miedos de estas víctimas les generan estrés y falta de concentración, disminuyendo así la precisión del recuerdo (Schaeffer et al., 2011). Por ello, es recomendable adaptar el proceso de declaración reduciendo la posibilidad de que las víctimas infantiles declaren bajo presión física o psicológica (Manzanero, 2010), con la intención de preservar el interés del menor y obtener la máxima calidad en el testimonio.

En España, esta variable es contemplada por la legislación en el apartado número 5 de la Circular N³/2009 de la Fiscalía General del Estado, sobre la protección de los menores víctimas y testigos, que establece como factores estresantes durante su declaración en el juicio oral:

- Permanecer solo en el lugar destinado a las víctimas.

- La proximidad del abogado defensor y la posibilidad de llegar a ser acusado de mentir.

- La posición en un lugar elevado de los actores del proceso.

- El público presente.

- Las togas de los intervinientes.

- El que se le exija hablar en alto.

- La utilización de un vocabulario legal ininteligible, especialmente cuando son examinados por el abogado de la defensa.

- La presencia del acusado.

A su vez, plantea adoptar una serie de medidas para contrarrestar estos factores estresantes: 
- Procurar que el menor sea el primero en declarar, evitando esperas en la puerta de la sala de juicios.

- Celebrar el juicio a puerta cerrada.

- Separar al menor del acusado, tomarle declaración en otra sala o evitar la visión directa con mamparas.

- Proporcionarle compañía durante la declaración (psicólogo o familiar), sobre todo para niños más pequeños.

- Utilizar un lenguaje comprensible.

- Suprimir el uso de togas durante la declaración del menor.

- Permitir que declaren sentados.

La Reforma $N^{\circ}$ 8/2006 aprobó el empleo de medios técnicos o audiovisuales, sin necesidad de informe pericial psicológico que lo motive, para evitar sobreexposición del menor a futuros interrogatorios, como la grabación previa de la entrevista del menor.

\section{iii. Sesgos: aquiescencia y familiaridad:}

Es importante señalar la tendencia de los preescolares a responder afirmativamente (sesgo de aquiescencia) ante preguntas disyuntivas perfectas que requieren confirmación o negación (si/no o verdadero-falso), tornando a un sesgo de respuesta negativa en menores de edad escolar, cuanto mayor sea la incomprensibilidad de la pregunta o el intervalo de demora (Fritzley et al., 2013, Mehrani y Peterson, 2017).

De igual modo, se debe considerar la posibilidad de basar erróneamente sus respuestas en la familiaridad o sensación subjetiva de probabilidad entre varias alternativas ante las preguntas de opción múltiple o elección forzosa (“¿La camisa era roja o azul?"), debiendo tener en cuenta que a mayor libertad en la elección de las alternativas de respuesta (preguntas abiertas, “¿De qué color era la camisa?"), más se reduce la probabilidad de aportar información falsa (Rocha et al., 2013).

\section{iv. Estimaciones erróneas:}

Los niños más pequeños pueden tener problemas al estimar cuantas veces ocurrió un suceso, ya que aprenden a recitar los números antes de que sepan contar objetos o eventos en su memoria. Por ello, el entrevistador deberá 
realizar un seguimiento por aproximación (p. ej., preguntando si ocurrió una o más veces, cuál fue la última y la primera vez, cual fue el episodio que mejor recuerda) (Saywitz et al., 2011)

También son comunes los problemas para establecer cuándo ocurrió un hecho, mejorando la cantidad y calidad de las referencias temporales con la edad (Orbach y Lamb, 2007). Para contrarrestar este déficit es útil emplear parámetros de comparación, como las referencias recientes de fechas destacadas para los niños (últimas vacaciones, navidades o cumpleaños) y los horarios de las rutinas diarias (comidas, clases o emisiones televisivas) (Orbach y Lamb, 2007).

\subsection{Procedimientos de entrevista}

En la última década, se ha trabajado en un protocolo de actuación para la conducción de esta prueba en colectivos vulnerables por parte de los profesionales técnicos, a partir de la experiencia práctica de psicólogos criminalistas y forenses (González et al., 2013). Los pasos propuestos a seguir por este protocolo son los siguientes:

- Vaciado de expediente y contacto con el contexto adulto del menor.

- Preparación del espacio físico.

- Preparación de la entrevista.

- Conducción de la entrevista.

Para realizar la entrevista a menores de edad se recomienda el protocolo NICHD (National Institute of Child Health and Human Development) (Lamb et al., 2007, citado González y Manzanero, 2018), en comparación con otros procedimientos de entrevista como el SATAC/RATAC (Anderson et al., 2010), que ha sido criticado por su sesgo confirmatorio y el uso de procedimientos sugestivos (Poole y Dickinson, 2011).

\section{i. Ambiente:}

El espacio donde se realice la exploración debe ser seguro, acogedor y confortable para la víctima (salas no muy grandes, luminosas y con la temperatura óptima), estar dotado de elementos neutros y relajantes (decoración informal) -evitando distraer a la víctima (no deben ser espacios de juego)—, con una apropiada disposición que no se interponga entre el entrevistador y el entrevistado y que permita ubicar a ambos a una distancia no muy lejana. 
A su vez, deberá ser silencioso para una tarea de escucha y grabación óptima, evitando posibles interrupciones (llamadas telefónicas, entrar en la sala otros profesionales o familiares, etc.). Para este fin, hace más de un siglo Arnold Gesell (1880-1961) diseñó las cámaras de observación que se conocen con su nombre para el estudio del desarrollo infantil. La cámara de Gesell facilitaba la observación de las exploraciones de los menores de edad con mínima interferencia y su grabación para el posterior estudio. Estos espacios se han propuesto como los lugares más adecuados para la realización de las entrevistas a víctimas especialmente vulnerables y para la pre-constitución o anticipación de la prueba. No obstante, a día de hoy, el desarrollo de la tecnología permite cumplir los mismos objetivos mediante video-conferencias o circuitos cerrados de televisión, que pueden sustituir las costosas cámaras Gesell que no están disponibles en todos los lugares.

En cualquier caso, el entrevistador deberá crear un ambiente que facilite la exposición de la mayor cantidad de información posible, para lo cual se personalizará la entrevista y se aportarán las instrucciones oportunas, minimizando el riesgo de falsos testimonios.

Teniendo en cuenta estas características, una opción es que el profesional se desplace al domicilio de la víctima, especialmente en víctimas menores de edad, en las cuales las dependencias policiales pueden sesgar su rendimiento de recuerdo.

\section{ii. Fase de introducción:}

Previamente a la obtención del testimonio del menor, es necesario obtener información acerca de su estado evolutivo y su desarrollo lingüístico, físico, social y sexual; ya sea en la propia entrevista con el niño o en entrevista con los adultos de referencia.

En la fase inicial o preparatoria (Saywitz et al., 2011.) es imprescindible la creación de una relación de colaboración entre el menor y el entrevistador. Teniendo en cuenta la indicación general en menores de edad de no hablar con extraños, antes de comenzar la exploración el entrevistador se presentará, explicará su función y la del menor, así como la finalidad indagatoria, promoviendo su cooperación, para lo cual podrá señalar la importancia de su declaración.

Se iniciará la conversación tratando temas neutros, por ejemplo, dialogando sobre los gustos del menor (juegos, comidas, asignaturas, etc.) (Bull, 2010, González y Manzanero, 2018), no sin antes haberle facilitado las instrucciones oportunas (p. ej., que debe exponer todo lo recordado, aunque le parezca poco 
relevante, y que cuando no sepa o no entienda algo podrá contestar "no lo sé" o "no lo entiendo"). Al respecto, una investigación actual (Canning y Peterson, 2020) sugiere que en niños de 3 a 4 años aumenta la cantidad de información proporcionada si se dan indicaciones precisas previas al recuerdo libre.

\section{iii. Fase de recuperación}

En esta fase se le invitará a una recuperación libre de la información con sus propias palabras, sin presiones, limitaciones temporales ni interrupciones, y evitando sugerir directamente el motivo de la exploración (Saywitz et al., 2011). Stolzenberg y Lyon (2017) observaron que, ante preguntas sobre la descripción y colocación de la ropa, las preguntas abiertas (p. ej., "¿dónde estaba la ropa?") llevaron a más detalles correctos que las preguntas de "Sí/No" y de elección forzada (p. ej., "¿llevabas puesta la ropa?”), pese a predominar estas últimas en la práctica judicial.

A esta fase le seguirá la formulación de preguntas dirigidas, cortas y de gramática simple con intención de precisar y ampliar su recuerdo, limitado por su desarrollo madurativo. Realizar indicaciones abiertas para incitar a que completen lo ya recordado (p. ej., "Me dijiste que - transcripción literal del menor- (...). Cuéntame más sobre eso") aumenta la precisión del recuerdo (Brown et al., 2013), la cantidad de información relevante evocada (Phillips et al., 2012), la coherencia y organización de las respuestas (Feltis et al., 2010) y la consistencia del relato, minimizando las contradicciones (Lamb y Fauchier, 2001).

Pese a ello, las investigaciones reflejan que se obtiene una mayor tasa de respuesta a indicaciones específicas que a abiertas en menores de 3 a 4 años (Hershkowitz et al., 2012). En este sentido, Wolfman, Brown y Jose (2016) estudiaron la capacidad de respuesta de víctimas de AS de 6 a 16 años en función del tipo de pregunta, y observaron que la probabilidad de obtener respuesta es mayor en las preguntas cerradas frente a las abiertas. Esto puede deberse a una mayor facilidad de respuesta percibida al requerir menor reflexión, aunque estas generen menor sensación de escucha (Brubacher et al., 2019).

En consecuencia, se ha de avanzar progresivamente desde preguntas abiertas a más específicas, evitando interrumpir o presionar al menor, dirigir sus respuestas, repetir insistentemente las mismas preguntas y preguntar la causa de algunos detalles (Manzanero y González, 2013), ya que esto podría generar falsos recuerdos, respuestas por deseabilidad social o autoatribución de la culpa. 
Asimismo, se evitará alterar el orden cronológico del suceso en la formulación de preguntas, ya que esto dificulta la localización de indicios adecuados para acceder a la memoria original, tomando como correcta la información sugerida (Paz-Alonso y Goodman, 2008).

Cabe señalar que las preguntas directas (p. ej., “¿Cómo te sentiste?”) son más útiles que las preguntas de recuerdo libre y las sugestivas en la obtención de contenido subjetivo, aumentando la capacidad de respuesta y la productividad de reacciones emocionales, contenido cognitivo y sensaciones físicas, siendo ventajoso integrar ambos tipos de preguntas en el interrogatorio (Lyon et al., 2012).

Sin embargo, las preguntas cerradas o sugerentes deben limitarse a circunstancias especiales, como hacer aclaraciones o a la valoración de la susceptibilidad ante información errónea, debido a la posibilidad de no contener la alternativa correcta o incluso añadir detalles falsos e inconsistencias (p. ej., la pregunta "¿De qué color era el perro?" supone la presencia de un perro en la escena) (Lamb y Fauchier, 2001). En consecuencia, el principal problema que plantea este tipo de preguntas es la imposibilidad de determinar cuándo la respuesta está basada en el recuerdo del testigo o víctima acerca del incidente, o en la conformidad o sumisión con la información proporcionada por el entrevistador.

\section{iv. Fase de cierre}

Se concluirá con una tercera fase o fase de cierre, destinando tiempo para realizar un resumen de lo expuesto, que el entrevistado plantee posibles dudas, agradecer su participación y ayudar a que se recomponga, y finalmente, desviar la entrevista hacia un tema neutro (Saywitz et al., 2011).

Como norma general, se realizarán descansos frecuentes para reducir la fatiga, se promoverá la grabación en vídeo de la entrevista, se limitará la presencia de otros adultos en la sala (incluidos los padres) y se evitará la prolongación de las sesiones en busca de una buena relación que garantice la no revictimización, ya que esto desorienta al menor de la finalidad principal de la exploración (Manzanero y González, 2013).

\subsection{Prácticas incorrectas en las entrevistas a menores de edad}

Bruck y Ceci (1995) listaron algunas de las prácticas de entrevista que pueden dar lugar a falsos recuerdos en casos de AS (Cuadro 1). 
1. Sesgo del entrevistador.

2. Repetición de preguntas.

3. Introducción de información falsa.

4. Tono emocional de los entrevistadores. Premios y castigos.

5. Influencia social "Otros me han dicho...".

6. Realización de la entrevista por un adulto de autoridad o estatus elevado.

7. Introducción de estereotipos.

8. Utilización de muñecos anatómicamente detallados, marionetas o dibujos.

9. Invitación a la especulación o imaginación.

\section{Cuadro 1.}

La buena praxis en la exploración de posibles víctimas de AS establece conocer el caso previamente a la realización de la entrevista, cuidando que estos conocimientos no supongan prejuicios que puedan provocar un sesgo confirmatorio en el afán por buscar explicaciones que confirmen las suposiciones creadas; valorando así positivamente los aspectos que la confirmen (Scott y Manzanero, 2015) y limitando la evocación del recuerdo original completo que permita interpretaciones alternativas (Ceci et al., 2007). El comportamiento no verbal del entrevistador (el tono de voz, los gestos o las expresiones faciales) también pueden sesgar las respuestas emitidas por los menores de edad (Almerigogna et al., 2008).

Como se ha adelantado en apartados anteriores, en el ámbito de la manipulación consciente o inconsciente del recuerdo infantil, las preguntas que guían el mismo son uno de los medios más comunes de sugestión en la exploración del menor, mereciendo una atención especial el aumento en la frecuencia de repetición de las preguntas durante las últimas décadas (Johnson et al., 2015). Andrews y Lamb (2014), en un estudio sobre la repetición de preguntas a presuntas víctimas menores de edad de AS, concluyeron que se repiten una media de 3 preguntas en cada entrevista, la mitad de éstas se realizan para cuestionar las respuestas ya aportadas o sin razón aparente (sin fines aclaratorios). En consecuencia, aproximadamente un tercio de los niños entrevistados elaboran 
una nueva respuesta a partir de la anterior y, cuando estas preguntas incluyen sugerencias, aumenta la probabilidad de generar auto-contradicciones entre las respuestas. Ese mismo año, otro estudio afirmaba al respecto que casi un 30\% de las respuestas de desconocimiento ("no sé") eran cuestionadas de nuevo aumentando el rigor de las preguntas (Earhart et al., 2014).

En definitiva, cuando se formulan las preguntas de forma clara y sin insistencia, la exactitud del recuerdo no difiere entre niños y adultos (Luus y Wells, 1992). De este modo, la presión resulta ser un mecanismo más poderoso en la sugestión interrogativa que la edad del entrevistado (Finnilä et al., 2003). Por ello, las preguntas deben repetirse únicamente en busca de aclaraciones, explicando el por qué y de forma abierta (Andrews y Lamb, 2014).

Por otro lado, los estereotipos inducidos durante la entrevista también acrecientan la sugestión (Blank et al., 2019), tornando la declaración de los menores de edad hacia información sugerida no neutral y generando falsos recuerdos. Lindberg, Keiffer y Thomas (2000), crearon un estereotipo en una muestra de menores de edad previo a la visualización de una escena sobre una disputa madre-hijo en la cual se escuchan golpes. Los menores de edad sesgados por el estereotipo de un hijo rebelde informaron que este derramó menos gotas de sangre ante una pregunta sugestiva sobre la agresión no visionada, frente a los menores de edad sesgados por el estereotipo de una madre con antecedentes de maltrato que informaron mayor cantidad de gotas de sangre. No obstante, el efecto sugestivo de los estereotipos inducidos durante la entrevista puede revertirse a posteriori si se advierte de la manipulación provocada por estos, posibilitándose así el recuerdo de la información original (Blank et al., 2019).

Los premios y castigos también actúan como mecanismo de sugestión, pudiendo incrementar el efecto de las preguntas sugestivas. Tal y como muestra la investigación, cuando se incluyen incentivos durante la exploración de menores de edad el porcentaje de falsas declaraciones se ve triplicado, se multiplica por 10 el efecto de las preguntas sugestivas fantasiosas y las falsas memorias se mantienen en entrevistas repetidas aun cuando el refuerzo es retirado (Garven et al., 2000). La formulación de preguntas abiertas contrarresta el efecto de los refuerzos aplicados en menores de edad, disminuyendo así la capacidad de manipular sus recuerdos autobiográficos y aumentando la precisión de sus testimonios (Hernández y Roebers, 2007). Una reciente investigación llevada a cabo por Rechdan, Sauer, Hope, Sauerland, Ost y Merckelbach (2017) sostiene que un feedback sobre su desempeño, en comparación con el de otros co-testigos, aumenta la aportación de detalles específicos en tareas de recuerdo libre. Sin embargo, ciertas expresiones de apoyo pueden ser sugestivas cuando se 
da retroalimentación positiva ante sugerencias erróneas, aumentando así las falsas memorias y la confianza en estas (Zaragoza et al., 2001).

Por último, la influencia social igualmente ejerce presión en la víctima, resultando una elevada probabilidad de aceptación de información no percibida, real o falsa, por una mayor confianza en los recuerdos ajenos (Selaya et al., 2020). En menores de edad esto ocurre comúnmente como consecuencia del sesgo de asentimiento por conformidad de pares; es decir, los niños cambian su negativa inicial cuando el entrevistador les informa que sus iguales manifestaron lo contrario, aumentando las tasas de asentimiento al verse influenciados por las sugerencias (Bruck et al., 2002). Sin embargo, en adultos, cuando la información errónea proviene de un co-testigo de avanzada edad, disminuye su confiabilidad y se reduce este efecto de conformidad (Thorley, 2015). En conclusión, la conformidad de la memoria por influencia social está determinada por la ausencia de recuerdos para esa información (Wright et al., 2005), por lo desagradables que resulten (Kieckhaefer y Wright, 2015) y por la precisión subjetiva otorgada a la memoria de la fuente (co-testigo, entrevistador) en relación con la propia (Williamson et al., 2013).

\section{ENTREVISTA A PERSONAS CON DISCAPACIDAD INTELECTUAL}

Como hemos dicho, otro colectivo susceptible de llegar a ser víctima de delitos contra la libertad e indemnidad sexual son las personas con DI. Esta condición implica limitaciones heterogéneas, entre las que está el funcionamiento intelectual y la conducta adaptativa (Manzanero et al., 2013.).

Se ha considerado erróneamente a este colectivo como problemático o violento, fantasioso, incapaces de tomar decisiones, de mentalidad infantil, insensibles al dolor, carentes de experiencia o deseo sexual y con conductas sexuales impulsivas y desadaptadas (Verdugo et al., 2002). Estas falsas creencias limitan la actuación del entrevistador en busca de las conclusiones correctas.

En primer lugar, si durante la recopilación de información se tiene sospecha de una posible discapacidad (pensamiento enlentecido, confusión, habilidades de comunicación reducidas, aquiescencia, etc.) y en ausencia de un documento o informe oficial que lo acredita, se ha de recurrir a profesionales expertos en la materia que puedan acreditar que dichas manifestaciones corresponden a una DI y no a cualquier otro trastorno mental (transitorio o no). En consecuencia, se realizará una evaluación psicológica a fin de determinar posibles afectaciones en la investigación. Asimismo, se aportarán pautas necesarias a los agentes policiales y judiciales sobre las adaptaciones pertinentes y apoyos 
que deban ser implementados en el procedimiento de toma de declaración y detección del AS.

Posteriormente, se valorará si se requiere de nuevo personal de apoyo independiente y especializado en DI y en Psicología del Testimonio durante el proceso policial o judicial (Recio et al., 2012) en respuesta a las necesidades de estas víctimas (informar sobre el funcionamiento del sistema policial y judicial, comprender las implicaciones y consecuencias judiciales, acompañar a la víctima durante el procedimiento, etc.), con el interés de que asesore con sus conocimientos a los agentes policiales y judiciales en la emisión de un juicio de credibilidad correcto, no basado en estereotipos y creencias, sino en las capacidades reales de la persona evaluada (Contreras et al., 2015).

Este colectivo resulta igualmente influenciable por los factores analizados anteriormente en el proceso de entrevista a menores de edad con desarrollo típico, aquellos factores que repercuten sobre la emisión de un relato basado en la evocación de un recuerdo original. Como ejemplo, se ha visto que la DI no parece establecer diferencias significativas respecto al colectivo de menores de edad en cuanto a la resistencia a preguntas sugestivas, incluso puede favorecer el rendimiento de la memoria en tareas de recuerdo libre (Henry y Gudjonsson, 2003). Sin embargo, en comparación con las víctimas menores de edad, parece que las características de las víctimas con DI plantean un reto en la búsqueda de información adicional ya codificada cuando se enfrentan a una tarea de recuerdo por indicios, dificultando, por ende, su rendimiento. Si bien, cabe destacar que, cuando se unen ambas condiciones, en menores de edad con DI resultan relatos libres menos completos y claros respecto a los menores de edad con desarrollo típico y, por consiguiente, menos creíbles (Henry et al., 2011), especialmente cuando no se practica una entrevista temprana (Brown et al., 2015). En cualquier caso, depende de cada tipo de discapacidad y de cada persona, ya que este colectivo es tremendamente heterogéneo.

En función del grado de discapacidad, mientras los menores de edad con DI leve son igual de capaces que sus iguales $\sin \mathrm{DI}$, en menores de edad con un grado severo de DI se observa un peor rendimiento y es necesario un contexto de apoyo para igualar las diferencias entre las competencias cognitivas (Brown et al., 2012). Pese a estos resultados, la práctica habitual en los interrogatorios policiales y judiciales es la formulación de preguntas cerradas y sugerentes, acrecentando la probabilidad de obtener información errónea (Cederborg y Lamb, 2008) y de contaminar sus declaraciones.

Por todo ello, además de las recomendaciones tratadas en el tercer apartado referentes al procedimiento de toma de declaración en menores de corta 
edad, González y Manzanero (2018) proponen la aplicación de las siguientes adaptaciones en las entrevistas a personas con DI:

- Escucha activa: mostrando empatía mediante expresiones no verbales, parafraseo, resúmenes, etc.

- Atención a la comunicación no verbal: favoreciendo la sensación de escucha y la concentración (p. ej., con un adecuado contacto visual).

- Paciencia: respetando los tiempos de respuesta y los silencios, y evitando corregirles o completar sus recuerdos.

- Preguntas abiertas: reduciendo la tendencia a responder de forma aquiescente por la gran deseabilidad social que les caracteriza, y limitando el uso de las preguntas cerradas de dos alternativas a casos de DI severa (siempre recordando la posibilidad de no saber o no acordarse).

- Claridad y simpleza en el lenguaje: utilizando un lenguaje comprensible, realizando una pregunta por frase, y evitando tecnicismos y construcciones complejas y extensas.

- Comprensión del relato: responsabilizándose a sí mismo de la falta de entendimiento pese a la contradicción o confusión del discurso de la víctima, y evitando formular repetidamente las mismas preguntas.

- Medios alternativos de comunicación: contrarrestando los problemas encontrados con los convencionales debido a sus limitaciones lingüísticas (p. ej., a través de gestos, dibujos, etc.).

\section{TESTIMONIO COMO PRUEBA PRECONSTITUIDA}

Bajo el derecho de ser protegidos, promoviendo su seguridad e intimidad y evitando su sufrimiento, el Estatuto de la Víctima del Delito propone como medida preventiva exponer a este tipo de víctimas a una única toma de declaración, grabada, que sirva como prueba pre-constituida de cara al juicio oral posterior (Caso et al., 2011). Con ello se evitan los efectos negativos que resultan de la intervención directa de policías, abogados defensores o fiscales no expertos en la materia (a través de preguntas cerradas, directivas, sugerentes, etc.) con víctimas de AS especialmente vulnerables. En suma, se salvaguarda el testimonio como indicio probatorio (de posibles alteraciones, errores 0 distorsiones de memoria), al reducir la sobreexposición de estos colectivos a repetidas evaluaciones y al consiguiente paréntesis temporal entre estas. De igual forma, se reduce la victimización secundaria (emociones negativas, sen- 
timiento de culpa, pensamientos de descrédito, etc.) comúnmente derivada de una inadecuada exposición a los procesos policiales y judiciales.

Su realización podrá ser motivada por el Juez de Instrucción o por un informe pericial psicológico y, en su defecto, promovida por el propio experto en consideración de la salvaguarda del desarrollo psicoevolutivo y la estabilidad psicológica de la víctima (Circular N³/2009 de Fiscalía General del Estado), siempre teniendo en cuenta aquellas limitaciones que puedan invalidar su práctica. Para ello, ha de realizarse un análisis previo del perfil de la víctima (capacidad lingüística, vulnerabilidad, resiliencia, desarrollo evolutivo, estado psicológico, etc.) y de las características del delito (frecuencia, duración, nivel de contacto físico, violencia aplicada, etc.). No obstante, la práctica de una prueba pre-constituida no exime a los profesionales de su deber de realizar el resto de las diligencias policiales y procesales oportunas en la evaluación forense del caso, como la investigación criminal de los supuestos hechos.

Teniendo en cuenta los dos objetivos fundamentales de la prueba pre-constituida: preservar el indicio cognitivo (testimonio) como elemento probatorio y evitar la victimización secundaria, se deben aplicar los protocolos existentes para la optimización de la obtención de las manifestaciones de la posible víctima (p. ej. el NICHD).

\section{AYUDAS AL RECUERDO}

Como hemos visto, la evocación de la memoria de los testigos y víctimas en ocasiones es inexacta o conlleva la omisión de detalles relevantes, por ende, los profesionales encargados de los procesos de toma de declaración pueden promover un mayor y mejor recuerdo mediante ayudas. Por ejemplo, los resultados derivados de las investigaciones acerca de los procedimientos de obtención del testimonio ponen de manifiesto que la recuperación con indicios mediante el empleo de señales sensoriales auditivas y visuales generan la activación de más representaciones semánticas y el recuerdo de más información autobiográfica (Karlsson et al., 2013.).

Asimismo, la reinstauración del entorno físico original en el cual se codificó la información puede facilitar la accesibilidad y el rendimiento en la tarea de recuerdo. Sin embargo, se ha demostrado que este procedimiento tiene el riesgo potencial de generar una nueva memoria en las víctimas que participan en la reconstrucción, que podría sustituir a la memoria original. Por consiguiente, si la reconstrucción no se ajusta exactamente a lo ocurrido puede dar lugar a declaraciones falsas. 
Por otro lado, con el interés de realizar una reconstrucción mental alejada de todo estímulo distractor, es común el gesto de cerrar los ojos ante tareas de recuerdo que exigen gran concentración. Diversos autores proponen que la instrucción de cerrar los ojos durante la evocación aumenta la cantidad de detalles aportados e inhibe el recuerdo de información falsa, gracias a la reducción de la carga de los recursos por las distracciones ambientales presentes (Perfect et al., 2008). No obstante, otras investigaciones con testigos reales han hallado que estos efectos son muy limitados, ya que no se obtenían mayores tasas de detalles recordados, sino que estos eran más relevantes y valiosos para la exploración forense y policial (Vredeveldt et al., 2015). Así, parece ser que el restablecimiento del contexto mental no aumenta la cantidad total de información recordada, sino que aumenta la cantidad de detalles recuperados libremente, mejorando la precisión del recuerdo especialmente en niños de edad preescolar y resultando más efectivo que la reinstauración del contexto físico (Hershkowitz et al., 2002).

Para finalizar, la probabilidad de acceso y recuperación de un recuerdo también depende de los indicios empleados en la búsqueda de la huella almacenada, de ahí la importancia de las instrucciones y del tipo de tarea empleada. A tal efecto, el NICHD (Lamb et al., 2007) constituye un elemento más completo y superior que la entrevista estándar; no obstante, la exactitud de la información obtenida mediante las entrevistas dependerá de la edad y perspectiva adoptada por el testigo.

\section{FALSAS AYUDAS AL RECUERDO}

A lo largo de la historia reciente se han propuesto otros procedimientos con el propósito de facilitar el recuerdo y la colaboración de los testigos o víctimas. Sin embargo, la investigación científica ha mostrado su escasa validez, ya que no logran alcanzar los objetivos propuestos. Concretamente, se desaconseja el uso forense de la hipnosis (Scoboria et al., 2008), las pruebas proyectivas (Wakefield y Underwager, 1993), los muñecos anatómicamente correctos (Bruck et al., 2000), la DRM (Zhu et al., 2013) y en general los procedimientos clínicos (Poole et al., 1995, Scott et al., 2014) para la exploración de las víctimas de AS, puesto que pueden aumentar la producción de falsas memorias.

En las entrevistas a víctimas de AS, dadas las características de los hechos delictivos vividos, es usual la narración de contacto físico entre agresor y víctima, generalmente de tocamientos, incluso en menores de corta edad. Procedimientos como el SATAC/RATAC (Anderson et al., 2010) han propuesto una serie de 
recursos para el proceso de toma de declaración, con el objetivo de facilitar la recuperación de aquellos detalles que no han sido especificados con las técnicas de obtención de información empleadas previamente, particularmente en víctimas con problemas de expresión como las personas con DI y los menores de corta edad. Sin embargo, estas pueden generar interpretaciones erróneas, por lo que han sido fuertemente criticadas.

\section{i. Dibujos y muñecos estándar:}

Se emplean con el propósito de facilitar el diálogo, fomentar la desenvoltura de la víctima y simplificar la complejidad de las situaciones delictivas de cara a su representación (p. ej., la distribución de espacios físicos y las personas que se encontraban en estos), y no para evaluar o interpretar lo representado. Es decir, no son métodos válidos para diagnosticar la ocurrencia de un delito sexual en la víctima, debido a la elevada probabilidad de falsas alarmas generadas al basar las conclusiones en interpretaciones erróneas (Köhnken et al., 2015; Scott et al., 2014). Por ello, su uso debe limitarse a aquellos niños de menor edad o personas con DI que presenten problemas de expresión, o en víctimas que presentan habilidades de comunicación, pero se requiere especificar una conducta dudosa (Manzanero y Barón, 2014).

\section{ii. Muñecos anatómicamente correctos:}

Este tipo de muñecos, dotados de atributos sexuales y características particulares de cada sexo (genitales, bello corporal, orificios, etc.) han sido creados para conocer las distintas partes corporales y representar conductas sexuales en contextos educativos. Su aplicación en contextos forenses puede generar una interpretación errónea de los hechos autobiográficos y, a la vez, distorsionar el recuerdo del menor, ya que pueden sugerir información falsa producto de la fantasía e imaginación infantil, por la preponderancia de ciertos detalles (Bruck et al., 2000, Thierry et al., 2006) o de la dificultad de usar los muñecos como una representación de ellos mismos (DeLoache y Marzolf, 1995).

Los resultados obtenidos por Bruck, Ceci y Hembrooke (2002) reflejan un aumento de los errores de comisión y omisión, así como de diferencias de género, encontrando más errores de comisión en niñas y de omisión en niños. Pese a ocasionar un aumento de la cantidad de detalles fantasiosos en menores de cualquier edad, se observa una mayor incidencia en niños de edad preescolar (Willcock et al., 2006), llegando incluso a contradecir los detalles proporcionados anteriormente (Thierry et al., 2006). 


\section{iii. Dibujos anatómicos:}

En cuanto a los dibujos de figuras humanas o diagramas corporales, resultan ventajosos para saber el grado de conocimientos que posee el entrevistado sobre las distintas partes del cuerpo y cómo éste suele denominarlas, aspecto especialmente útil de cara a la formulación de las preguntas en un lenguaje que no sesgue el recuerdo de la víctima.

Sin embargo, la investigación practicada desaconseja su uso para ubicar los tocamientos y especialmente en los niños más pequeños (menores de 5 años), ya que, pese a recabar mayor cantidad de información sobre el contacto, se reduce la exactitud del testimonio, al informar de nuevos falsos contactos y elaboraciones relacionadas con aquellas zonas corporales supuestamente tocadas (Aldridge et al., 2004; Brown, et al. , 2007; Poole y Dickinson, 2011; y Willcock et al., 2006).

En la misma línea, la investigación llevada a cabo por Aldridge, Lamb, Sternberg, Orbach, Esplin y Bowler en 2004 concluyó que el aumento de la información aportada con el empleo de estos dibujos disminuye con la edad del menor; recomendándose, por ello, la introducción tardía en el proceso de investigación y el empleo de indicaciones específicas, no sugestivas, que promuevan el recuerdo libre tras las respuestas afirmativas.

Por consiguiente, estos métodos de apoyo a la entrevista forense no gozan de evidencias acerca de la mejora de la capacidad de informar contactos físicos en los niños de corta edad o personas con DI, y sí de un alto riesgo de errores, por lo que deben explorarse otros métodos para la obtención de las revelaciones de tocamientos relacionados con prácticas sexuales (Poole et al., 2011). En definitiva, el uso de ayudas no verbales (juegos, dibujar, y muñecos o dibujos anatómicos) y procedimientos directivos de entrevista no resultan producentes para la revelación de contacto físico, ni aun respetando las recomendaciones profesionales (Salmon et al., 2012).

Respecto a realizar dibujo libre durante la entrevista, parece que no afecta a la precisión y cantidad en las tareas de recuerdo libre (Poole y Dickinson, 2014), e incluso puede aumentar la integridad del recuerdo y la cantidad de detalles, aunque sí favorece la disminución de la precisión del testimonio para eventos complejos (Otgaar et al., 2016), ante la inclusión de preguntas falsas (Bruck et al., 2000) y, tras largas demoras (Salmon y Pipe, 2000), potenciando más aún los efectos sugestivos de estos factores.

En consecuencia, pese a la existencia de técnicas que pueden mejorar la cantidad de información recuperada, algunos entrevistadores desconocen en 
qué condiciones y momentos resultan efectivas y, en ese caso, han de abstenerse de aplicarlas por la posible sugestión derivada de su mal uso (Pipe et al., 2004).

\section{CONCLUSIONES}

Las víctimas de corta edad y/o personas con DI pueden ser informantes valiosos cuando son explorados por profesionales que reconocen sus limitaciones y fortalezas y adaptan la práctica de la entrevista a sus características.

En el proceso de toma de declaración, los falsos testimonios pueden provenir de dos fuentes básicas: la mentira, por omisión o distorsión deliberada de los hechos, o las falsas memorias, recuerdos inexactos que difieren parcial o totalmente del recuerdo original debido al olvido o a la distorsión no deliberada de la memoria inicial. Asimismo, son numerosos los factores que influyen en la codificación, retención y recuperación de la información, haciendo vulnerable al recuerdo exacto de unos hechos autobiográficos y pudiendo generarse una falsa memoria.

En la generación de falsas memorias, adquiere suma importancia el formato de recuperación, en concreto, las preguntas durante los interrogatorios y el manejo de la entrevista policial (Shaw y Porter, 2015). Así, las palabras empleadas en las preguntas sobre los detalles del suceso sesgan la intensidad y la probabilidad de ocurrencia de lo preguntado. El procedimiento de obtención de la declaración puede alterar la representación mental que el entrevistado tenga de los hechos, deteriorar la concentración e introducir elementos sugestivos. Por ello, se han propuesto diferentes técnicas de interrogatorio como la Entrevista Cognitiva (EC) (Fisher y Geiselman, 2010), y procedimientos de entrevista específicos para menores de edad como el protocolo NICHD (Lamb et al., 2007, González y Manzanero, 2018).

La relevancia de las falsas memorias en el ámbito judicial se sustenta en las implicaciones de éstas en la memoria de testigos y en las posibles repercusiones o medidas adoptadas erróneamente ante la verbalización distorsionada de los hechos vivenciados por esta víctima o testigo, resultando una falsa condena o la absolución de un culpable.

Uno de los inconvenientes de generar una falsa memoria es la dificultad de convencer posteriormente al sujeto de que realmente sus recuerdos sufrieron una transformación por la información falsa post-suceso. Otro inconveniente es su estabilidad en el tiempo, cuanto mayor sea la demora de su recuerdo, mayor será el arraigo de la huella y, en consecuencia, mayor será la seguridad 
de haberlo vivenciado tal y como falsamente recuerdan. Por otro lado, la imposibilidad de los testigos de inhibir voluntariamente una falsa memoria previamente establecida y, yendo más allá, el esfuerzo por olvidar esa huella puede producir un efecto rebote, convirtiéndola en un pensamiento intrusivo (Pitarque et al., 2003).

Durante la obtención del testimonio existen varios factores que influyen en la recuperación de la información almacenada en nuestra memoria y que deberemos controlar si queremos mejorar el rendimiento del recuerdo. Entre ellos, el entrevistador puede ser una fuente sugestiva igual de potente o más que otras fuentes sociales como los co-testigos (Jack et al., 2014), pudiendo deberse a la mayor confianza depositada en ellos, dada su experiencia y autoridad. Pero, además de los factores asociados al entrevistador, es necesario evaluar los factores asociados a la supuesta víctima (desarrollo evolutivo, capacidad cognitiva, etc.) porque solo así podremos adaptar la entrevista a sus capacidades, facilitando la recuperación. No podemos olvidar que las características del contenido del relato gozan de mayor validez en la posterior evaluación de la credibilidad cuando el relato es obtenido de forma abierta, no sugerente, sin demora y adaptándose a las características de la víctima.

\section{LISTA DE REFERENCIAS}

Aldridge, J., Lamb, M., Sternberg, K., Orbach, Y., Esplin, P. y Bowler, L. (2004). Using a human figure drawing to elicit information from alleged victims of child sexual abuse. Journal of Consulting and Clinical Psychology, 72(2), 304-316. https://doi.org/10.1037/0022-006X.72.2.304

Alemany, A., Quintana, J., Recio, M., Silva, E., Manzanero, A., Martorell, A., y González, J. (2012). Guía de intervención policial con personas con discapacidad intelectual. Fundación Carmen Pardo-Valcarce.

Almerigogna, J., Ost, J., Akehurst, L. y Fluck, M. (2008). How interviewers' nonverbal behaviors can affect children's perceptions and suggestibility. Journal of Experimental Child Psychology, 100(1), 17-39. https://doi.org/10.1016/j. jecp.2008.01.006

Anderson, J., Ellefson, J., Lashley, J., Miller, A., Olinger, S., Russell, A., Stauffer, J., y Weigman, J. (2010). The Cornerhouse Forensic Interview Protocol: RATAC. Cooley Journal of Practice and Clinical Law, 1, 193-331.

Andrews, S. y Lamb, M. (2014). The effects of age and delay on responses to repeated questions in forensic interviews with children alleging sexual abuse. Law and Human Behavior, 38(2), 171-180. https://doi.org/10.1037//hb0000064r 
Antaki, C., Richardson, E., Stokoe, E. y Willott, S. (2015). Can people with intellectual disability resist implications of fault when police question their allegations of sexual assault and rape? Intellectual and Developmental Disabilitie, 53(5), 346-357. https://doi.org/10.1352/1934-9556-53.5.346

Baugerud, G., Johnson, M., Hansen, H., Magnussen, S. y Lamb, M. (2020). Forensic interviews with preschool children: An analysis of extended interviews in Norway (2015-2017). Applied Cognitive Psychology, 34(3), 654-663. https:// doi.org/10.1002/acp.3647

Bjorklund, D., Cassel, W., Bjorklund, B., Brown, R., Park, C., Ernst, K. y Owen, F. (2000). Social demand characteristics in children's and adults' eyewitness memory and suggestibility: The effect of different interviewers on free recall and recognition. Applied Cognitive Psychology, 14(5), 421-433. https://doi. org/10.1002/1099-0720(200009)14:5<421::AID-ACP659>3.0.CO;2-4

Blank, H., Rutter, L. y Armstrong, R. (2019). Enlightenment beats prejudice: The reversibility of stereotype-induced memory distortion. Psychonomic Bulletin and Review, 26(3), 1001-1007. https://doi.org/10.3758/s13423-018-1541-7

Blasbalg, U., Hershkowitz, I., Lamb, M., Karni-Visel, Y. y Ahern, E. (2019). Is interviewer support associated with the reduced reluctance and enhanced informativeness of alleged child abuse victims? Law and Human Behavior, 43(2), 156-165. https://doi.org/10.1037//hb0000303

Brown, D. y Lamb, M. (2015). Can children be useful witnesses? It depends how they are questioned. Child Development Perspectives, 9(4), 250-255. https:// doi.org/10.1111/cdep.12142

Brown, D., Lamb, M., Lewis, C., Pipe, M.-E., Orbach, Y. y Wolfman, M. (2013). Evaluating the NICHD investigative interview protocol: An analogue study. Journal of Experimental Psychology: Applied, 19(4), 367-382. https://doi. org/10.1037/a0035143

Brown, D., Lewis, C. y Lamb, M. (2015). Preserving the past: An early interview improves delayed event memory in children with intellectual disabilities. Child Development, 86(4), 1031-1047. https://doi.org/10.1111/cdev.12364

Brown, D., Lewis, C., Lamb, M. y Stephens, E. (2012). The influences of delay and severity of intellectual disability on event memory in children. Journal of Consulting and Clinical Psychology, 80(5), 829-841. https://doi.org/10.1037/ a0029388

Brown, D., Pipe, M., Lewis, C., Lamb, M. y Orbach, Y. (2007). Supportive or suggestive: Do human figure drawings help 5 to 7 -year-old children to report touch? Journal of Consulting and Clinical Psychology, 75(1), 33-42. https:// doi.org/10.1037/0022-006X.75.1.33 
Brubacher, S., Timms, L., Powell, M. y Bearman, M. (2019). "She Wanted to Know the Full Story": Children's Perceptions of Open Versus Closed Questions. Child Maltreatment, 24(2), 222-231. https://doi.org/10.1177/1077559518821730

Bruck, M. y Ceci, S. (1995). Amicus brief for the case of State of New Jersey v. Michaels presented by Committee of Concerned Social Scientists. Psychology, Public Policy, and Law, 1(2), 272-322. https://doi.org/10.1037/1076-8971.1.2.272

Bruck, M., Ceci, S. y Francoeur, E. (2000). Children's use of anatomically detailed dolls to report genital touching in a medical examination: Developmental and gender comparisons. Journal of Experimental Psychology: Applied, 6(1), 74-83. https://doi.org/10.1037/1076-898X.6.1.74

Bruck, M., Ceci, S., Francouer, E. y Renick, A. (1995). Anatomically detailed dolls do not facilitate preschoolers' reports of a pediatric examination involving genital touching. Journal of Experimental Psychology: Applied, 1(2), 95-109. https://doi.org/10.1037/1076-898X.1.2.95

Bruck, M., Ceci, S. y Hembrooke, H. (2002). The nature of children's true and false narratives. Developmental Review, 22(3), 520-554. https://doi.org/10.1016/ S0273-2297(02)00006-0

Bruck, M., Melnyk, L. y Ceci, S. (2000). Draw it again Sam: The effect of drawing on children's suggestibility and source monitoring ability. Journal of Experimental Child Psychology, 77(3), 169-196. https://doi.org/10.1006/jecp.1999.2560

Bull, R. (2010). The investigative interviewing of children and other vulnerable witnesses: Psychological research and working/professional practice. Legal and Criminological Psychology, 15, 5-23. https://doi.or$\mathrm{g} / 10.1348 / 014466509 \times 440160$

Canning, H. y Peterson, C. (2020). Encouraging more open-ended recall in child interviews. Psychiatry, Psychology and Law, 27(1), 81-94. https://doi.org/1 0.1080/13218719.2019.1687045

Caso, M., Arch, M., Jarne, A. y Molina, A. (2011). Guía práctica de exploración de menores. Sepín.

Ceci, S., Kulkofsky, S., Klemfuss, J., Sweeney, C. y Bruck, M. (2007). Unwarranted assumptions about children's testimonial accuracy. Annual Review of Clinical Psychology, 3(1), 311-328. https://doi.org/10.1146/annurev. clinpsy.3.022806.091354

Cederborg, A. y Lamb, M. (2008). Interviewing alleged victims with intellectual disabilities. Journal of Intellectual Disability Research, 52(1), 49-58.

Cederborg, A. y Hellner, C. (2010). The challenge of assessing credibility when children with intellectual disabilities are alleged victims of abuse. Scan- 
dinavian Journal of Disability Research, 12(2), 125-140. https://doi. org/10.1080/15017410902909134

Chrobak, Q., Rindal, E. y Zaragoza, M. (2015). The impact of multifaceted questions on eyewitness accuracy following forced fabrication interviews. Journal of General Psychology, 142(3), 150-166. https://doi.org/10.1080/00221309. 2015.1060188

Contreras, M., Silva, E. y Manzanero, A. (2015). Evaluación de capacidades para testificar en víctimas con discapacidad intelectual. Anuario de Psicología Jurídica, 25, 87-96. https://doi.org/10.1016/j.apj.2015.02.006

DeLoache, J. y Marzolf, D. (1995). The use of dolls to interview young children: Issues of symbolic representation. Journal of Experimental Child Psychology, 60(1), 155-173. https://doi.org/10.1006/jecp.1995.1036

Earhart, B., La Rooy, D., Brubacher, S., y Lamb, M. (2014). An examination of "don't know" responses in forensic interviews with children. Behavioral Sciences and the Law, 32(6), 746-761. https://doi.org/10.1002/bsl.2141

Feltis, B., Powell, M., Snow, P. y Hughes-Scholes, C. (2010). An examination of the association between interviewer question type and story-grammar detail in child witness interviews about abuse. Child Abuse and Neglect, 34(6), 407-413. https://doi.org/10.1016/j.chiabu.2009.09.019

Finnilä, K., Mahlberg, N., Santtila, P., Sandnabba, K. y Niemi P. (2003). Validity of a test of children's suggestibility for predicting responses to two interview situations differing in their degree of suggestiveness. Journal of Experimental Child Psychology, 85(1), 32-49. https://doi.org/10.1016/S0022-0965(03)00025-0

Fisher, R. y Geiselman, R. (2010). The Cognitive Interview method of conducting police interviews: Eliciting extensive information and promoting Therapeutic Jurisprudence. International Journal of Law and Psychiatry, 30 (5-6), 321328. https://doi.org/10.1016/j.ijlp.2010.09.004

Foster, J. (2017). It Happened to Me: A Qualitative Analysis of Boys' Narratives About Child Sexual Abuse. Journal of Child Sexual Abuse, 26(7), 853-873. https://doi.org/10.1080/10538712.2017.1360426

Fritzley, V., Lindsay, R. y Lee, K. (2013). Young children's response tendencies toward yes-no questions concerning actions. Child Development, 84(2), 711-725. https://doi.org/10.1111/cdev.12006

Garven, S., Wood, J. y Malpass, R. (2000). Allegations of wrongdoing: The effects of reinforcement on children's mundane and fantastic claims. Journal of Applied Psychology, 85(1), 38-49. https://doi.org/10.1037/0021-9010.85.1.38 
González, J., Cendra, J. y Manzanero, A. (2013). Prevalence of disabled people involved in Spanish Civil Guard's police activity. Research in Developmental Disabilities, 34(11), 3781-3788. https://doi.org/10.1016/j.ridd.2013.08.003

González, J. y Manzanero, A. (2018). Obtención y valoración del testimonio. Protocolo Holístico de Evaluación de la Prueba Testifical (HELPT). Pirámide.

González, J., Muñoz, J., Sotoca, A. y Manzanero, A. (2013). Propuesta de protocolo para la conducción de la prueba preconstituida en víctimas vulnerables. Papeles del Psicólogo, 34(3), 227-237.

Gurney, D., Pine, K. y Wiseman, R. (2013). The Gestural Misinformation Effect: Skewing Eyewitness Testimony Through Gesture. The American Journal of Psychology, 126(3), 301-314. https://doi.org/10.5406/amerjpsyc.126.3.0301

Henry, L. y Gudjonsson, G. (2003). Eyewitness memory, suggestibility and repeated recall sessions in children with mild and moderate intellectual disabilities. Law and Human Behavior, 27(5), 481-505. https://doi.org/10.1023/A:1025434022699

Henry, L., Ridley, A., Perry, J. y Crane, L. (2011). Perceived credibility and eyewitness testimony of children with intellectual disabilities. Journal of Intellectual Disability Research, 55(4), 385-391. https://doi.org/10.1111/ j.1365-2788.2011.01383.x

Hernández, C. y Roebers, C. (2007). Efectos de los incentivos en la regulación estratégica de los testimonios infantiles. Anuario de Psicología, 38(3), 413-430.

Hershkowitz, I. y Lamb, M. (2020). Allegation rates and credibility assessment in forensic interviews of alleged child abuse victims: Comparing the revised and standard NICHD protocols. Psychology, Public Policy, and Law, 26(2), 176-184. https://doi.org/10.1037/law0000230

Hershkowitz, I., Lamb, M., Orbach, Y., Katz, C. y Horowitz, D. (2012). The development of communicative and narrative skills among preschoolers: lessons from forensic interviews about child abuse. Child Development, 83(2), 611622. https://doi.org/10.1111/j.1467-8624.2011.01704.x

Hershkowitz, I., Orbach, Y., Lamb, M., Sternberg, K. y Horowitz, D. (2002). A comparison of mental and physical context reinstatement in forensic interviews with alleged victims of sexual abuse. Applied Cognitive Psychology, 16(4), 429-441. https://doi.org/10.1002/acp.804

Jack, F., Zydervelt, S. y Zajac, R. (2014). Are co-witnesses special? Comparing the influence of co-witness and interviewer misinformation on eyewitness reports. Memory, 22(3), 243-255. https://doi.org/10.1080/09658211.201 3.778291 
Johnson, M., Magnussen, S., Thoresen, C., Lønnum, K., Burrell, L. y Melinder, A. (2015). Best practice recommendations still fail to result in action: a national 10-year follow-up study of investigative interviews in CSA cases. Applied Cognitive Psychology, 29(5), 661-668. https://doi.org/10.1002/acp.3147

Karlsson, K., Sikström, S. y Willander, J. (2013). The semantic representation of event information depends on the cue modality: an instance of meaningbased retrieval. PloS one, 8(10), e73378. https://doi.org/10.1371/journal. pone. 0073378

Kieckhaefer, J. y Wright, D. (2015). Likable co-witnesses increase eyewitness accuracy and decrease suggestibility. Memory, 23(3), 462-472. https://doi.or $\mathrm{g} / 10.1080 / 09658211.2014 .905607$

Köhnken, G., Manzanero, A. y Scott, M. (2015). Análisis de la validez de las declaraciones: mitos y limitaciones. Anuario de Psicología Jurídica, 25(1), 13-19. https://doi.org/10.1016/j.apj.2015.01.004

Lamb, M. y Brown, D., (2006). Conversational apprentices: Helping children become competent informants about their own experiences. The British Journal of Developmental Psychology, 24(1), 215-234. https://doi. org/10.1348/026151005X57657

Lamb, M. E. y Fauchier, A. (2001). The effects of question type on self-contradictions by children in the course of forensic interviews. Applied Cognitive Psychology, 15(5), 483-491. https://doi.org/10.1002/acp.726

Lamb, M., Orbach, Y., Hershkowitz, I., Esplin, P. y Horowitz, D. (2007). A structured forensic interview protocol improves the quality and informativeness of investigative interviews with children: A review of research using the NICHD Investigative Interview Protocol. Child Abuse and Neglect, 31(11-12), 12011231. https://doi.org/10.1016/j.chiabu.2007.03.021

Lamb, M., Sternberg, K. J., Orbach, Y., Hershkowitz, I. y Horowitz, D. (2003). Differences between accounts provided by witnesses and alleged victims of child sexual abuse. Child Abuse and Neglect, 27(9), 1019-1031. https://doi. org/10.1016/S0145-2134(03)00167-4

Lane, S., Mather, M., Villa, D. y Morita, S. (2001). How events are reviewed matters: effects of varied focus on eyewitness suggestibility. Memory and Cognition, 29 (7), 940-947. https://doi.org/10.3758/BF03195756

Lindberg, M., Keiffer, J. y Thomas, S. (2000). Eyewitness testimony for physical abuse as a function of personal experience, development, and focus of study. Journal of Applied Developmental Psychology, 21(5), 555-591. https://doi. org/10.1016/S0193-3973(00)00054-X 
Luus, E. y Wells, G. (1992). The perceived credibility of child eyewitnesses. En H. Dent y R. Flin (eds.), Children as witnessess. Wiley.

Lyon, T., Scurich, N., Choi, K., Handmaker, S. y Blank, R. (2012). "How did you feel?": increasing child sexual abuse witnesses' production of evaluative information. Law and Human Behavior, 36(5), 448-457. https://doi.org/10.1037/ h0093986

Manzanero, A. (2010). Memoria de Testigos: Obtención y valoración de la prueba testifical. Pirámide.

Manzanero, A. y Barón, S. (2014). Características de las memorias en niños preescolares: obtención y evaluación de sus recuerdos. En M. Meriño (coord.) Los delitos sexuales desde una perspectiva interdisciplinaria (pp. 51-83). Ediciones Jurídicas de Santiago.

Manzanero, A., Contreras, M., Recio, M., Alemany, A. y Martorell, A. (2012). Effects of presentation format and instructions on the ability of people with intellectual disability to identify faces. Research in developmental disabilities, 33(2), 391-397. https://doi.org/10.1016/j.ridd.2011.09.015

Manzanero, A. y González, J. (2013). Avances en Psicología del Testimonio. Ediciones Jurídicas de Santiago.

Manzanero, A., Recio, M., Alemany, A., y Cendra, J. (2013). Atención a víctimas con discapacidad intelectual. Fundación Carmen Pardo-Valcarce.

Martorell, A. y Alemany, A. (2017). La figura del facilitador en casos de abuso sexual a personas con discapacidad intelectual. Siglo Cero, 48(261), 37-49. https:// doi.org/10.14201/scero2017483749

McFarlane, F., Powell, M. y Dudgeon, P. (2010). An examination of the degree to which IQ, memory performance, socio-economic status and gender predict young children's suggestibility. Legal and Criminological Psychology, 7(2), 227-239. https://doi.org/10.1348/135532502760274729

Mehrani, M. y Peterson, C. (2017). Responses to interview questions: A cross-linguistic study of acquiescence tendency. Infant and Child Development, 27(2), e2063. https://doi.org/10.1002/icd.2063

Milne, R. y Bull, R. (2006). Interviewing victims of crime, including children and people with intellectual disabilities. En M. Kebbell y G. Davies (eds.), Practical Psychology for Forensic Investigations (pp. 7-24). Wiley. https://doi. org/10.1002/9780470713389.ch1

Orbach, Y. y Lamb, M. (2007). Young children's references to temporal attributes of allegedly experienced events in the course of forensic interviews. 
Child Development, 78(4), 1100-1120. https://doi.org/10.1111/j.14678624.2007.01055.x

Otgaar, H., van Ansem, R., Pauw, C. y Horselenberg, R. (2016). Improving children's interviewing methods? the effects of drawing and practice on children's memories for an event. Journal of Police and Criminal Psychology, 31(4), 279-287. https://doi.org/10.1007/s11896-016-9190-0

Paz-Alonso, P. y Goodman, G. (2008). Trauma and memory: Effects of post-event misinformation, retrieval order, and retention interval. Memory, 16(1), 5875. https://doi.org/10.1080/09658210701363146

Perfect, T., Wagstaff, G., Moore, D., Andrews, B., Cleveland, V., Newcombe, S., Brisbane, K.-A. y Brown, L. (2008). How can we help witnesses to remember more? It's an (eyes) open and shut case. Law and Human Behavior, 32(4), 314-324. https://doi.org/10.1007/s10979-007-9109-5

Peterson, C. y Bell, M. (1996). Children's memory for traumatic injury. Child Development, 67(6), 3045-3070. https://doi.org/10.2307/1131766

Peterson, C., Hallett, D. y Compton-Gillingham, C. (2018). Childhood amnesia in children: A prospective study across eight years. Child Development, 89(6), e520-e534. https://doi.org/10.1111/cdev.12972

Peterson, C., Warren, K. y Short, M. (2011). Infantile amnesia across the years: a 2-year follow-up of children's earliest memories. Child Development, 82(4), 1092-1105. https://doi.org/10.1111/j.1467-8624.2011.01597.x

Phillips, E., Oxburgh, G., Gavin, A. y Myklebust, T. (2012). Investigative interviews with victims of child sexual abuse: The relationship between question type and investigation relevant information. Journal of Police and Criminal Psychology, 27(1), 45-54. https://doi.org/10.1007/s11896-011-9093-z

Pipe, M., Lamb, M., Orbach, Y. y Esplin, P. (2004). Recent research on children's testimony about experienced and witnessed events. Developmental Review, 24(4), 440-468. https://doi.org/10.1016/j.dr.2004.08.006

Pitarque, A., Algarabel, S., Dasí, C. y Ruiz, J. (2003). Olvido dirigido de falsas memorias: ¿ंPodemos olvidar intencionadamente una falsa memoria? Psicothema, 15(1), 6-11.

Poole, D., Bruck, M. y Pipe, M. (2011). Forensic Interviewing Aids: Do Props Help Children Answer Questions About Touching? Current Directions in Psychological Science, 20(1), 11-15. https://doi.org/10.1177/0963721410388804

Poole, D. y Dickinson, J. (2011). Evidence supporting restrictions on uses of body diagrams in forensic interviews. Child Abuse and Neglect, 35(9), 659-669. https://doi.org/10.1016/j.chiabu.2011.05.004 
Poole, D. y Dickinson, J. (2014). Comfort drawing during investigative interviews: Evidence of the safety of a popular practice. Child Abuse and Neglect, 38(2), 192-201. https://doi.org/10.1016/j.chiabu.2013.04.012

Poole, D., Lindsay, D., Memon, A. y Bull, R. (1995). Psychotherapy and the recovery of memories of childhood sexual abuse: US and British practitioners' opinions, practices, and experiences. Journal of Consulting and Clinical Psychology, 63(3), 426. https://doi.org/10.1037/0022-006X.63.3.426

Rechdan, J., Sauer, J., Hope, L., Sauerland, M., Ost, J. y Merckelbach, H. (2017). Computer mediated social comparative feedback does not affect metacognitive regulation of memory reports. Frontiers in Psychology, 8, 1433. https:// doi.org/10.3389/fpsyg.2017.01433

Recio, M., Alemany, A. y Manzanero, A. (2012). La figura del facilitador en la investigación policial y judicial con víctimas con discapacidad intelectual. Siglo Cero, 43(3), 54-68.

Rocha, E., Marche, T. y Briere, J. (2013). The effect of forced-choice questions on children's suggestibility: A comparison of multiple-choice and yes/no questions. Canadian Journal of Behavioural Science, 45(1), 1-11. https:// doi.org/10.1037/a0028507

Salmon, K. y Pipe, M. (2000). Recalling an event one year later: The impact of props, drawing and a prior interview. Applied Cognitive Psychology, 14(2), 99-120. https://doi.org/10.1002/(SICI)1099-0720(200003/04)14:2<99::AI D-ACP639>3.0.CO;2-5

Salmon, K., Pipe, M., Malloy, A. y Mackay, K. (2012). Do nonverbal aids increase the effectiveness of 'best practice' verbal interview techniques? An experimental study. Applied Cognitive Psychology, 26(3), 370-380.

Saywitz, K., Lyon, T. y Goodman, G. (2011). Interviewing children. En J. E. B. Myers (ed.). APSAC handbook on child maltreatment (3.a ed) (pp. 337-360). Sage Publications.

Saywitz, K., Wells, C., Larson, R. y Hobbs, S. (2019). Effects of interviewer support on children's memory and suggestibility: systematic review and meta-analyses of experimental research. Trauma, Violence and Abuse, 20(1), 22-39. https:// doi.org/10.1177/1524838016683457

Schaeffer, P., Leventhal, J. y Asnes, A. (2011). Children's disclosures of sexual abuse: learning from direct inquiry. Child Abuse and Neglect, 35(5), 343-352. https:// doi.org/10.1016/j.chiabu.2011.01.014

Selaya, A., Sanmarco, J. y Arce, R. (2020). Estimación de la conformidad en el testimonio: una revisión meta-analítica. En A. M. Martín, F. Fariña y R. Arce (eds.), 
Psicología jurídica y forense: investigación para la práctica professional (pp. 69-80). Sociedad Española de Psicología Jurídica y Forense.

Scoboria, A., Mazzoni, G., y Kirsch, I. (2008). "Don't know" responding to answerable and unanswerable questions during misleading and hypnotic interviews. Journal of Experimental Psychology: Applied, 14(3), 255-265. https://doi. org/10.1037/1076-898X.14.3.255

Scott, M. y Manzanero, A. (2015). Análisis del expediente judicial: Evaluación de la validez de la prueba testifical. Papeles del Psicólogo, 36(2), 139-144.

Scott, M., Manzanero, A., Muñoz, J., y Köhnken, G. (2014). Admisibilidad en contextos forenses de indicadores clínicos para la detección del abuso sexual infantil. Anuario de Psicología Jurídica, 24(1), 57-63. https://doi. org/10.1016/j.apj.2014.08.001

Seoane, J. (2004). Derecho y personas con discapacidad. Hacia un nuevo paradigma. Siglo Cero, 35(1), 20-50.

Shaw, J. y Porter, S. (2015). Constructing rich false memories of committing crime. Psychological Science, 26(3), 291-301. https://doi. org/10.1177/0956797614562862

Silva, E., Manzanero, A. y Contreras, M. (2018). CAPALIST. Valoración de capacidades para testificar. Dykinson.https://doi.org/10.2307/j.ctv9zcgtc

Stolzenberg, S. y Lyon, T. (2017). 'Where were your clothes?' Eliciting descriptions of clothing placement from children alleging sexual abuse in criminal trials and forensic interviews. Legal and Criminological Psychology, 22(2), 197-212. https://doi.org/10.1111/lcrp.12094

Teoh, Y. y Lamb, M. (2013). Interviewer demeanor in forensic interviews of children. Psychology, Crime and Law, 19(2), 145-159. https://doi.or$\mathrm{g} / 10.1080 / 1068316 X .2011 .614610$

Thierry, K., Lamb M., Orbach, Y. y Pipe, M. (2006). Developmental differences in the function and use of anatomical dolls during interviews with alleged sexual abuse victims. Journal of Consulting and Clinical Psychology, 73(6), 1125-1134. https://doi.org/10.1037/0022-006X.73.6.1125

Thorley, C. (2015). Blame conformity: innocent bystanders can be blamed for a crime as a result of misinformation from a young, but not elderly, adult co-witness. PloS one, 10(7), e0134739. https://doi.org/10.1371/journal. pone.0134739

Vallet, R., Manzanero, A., Aróztegui, J., y García-Zurdo, R. (2017). Age-related differences in phenomenal characteristics of long-term memories for the attack 
of March 11, 2004. Anuario de Psicología Jurídica, 27(1), 85-93. https://doi. org/10.1016/j.apj.2017.03.002

Verdugo, M., Alcedo, M., Bermejo, B. y Aguado, A. (2002). Abuso sexual en personas con discapacidad intelectual. Psicothema, 14, supl, 124-129.

Volpini, L., Melis, M., Petralia, S. y Rosenberg, M. (2016). Measuring Children's Suggestibility in Forensic Interviews. Journal of Forensic Sciences, 61(1), 104-108. https://doi.org/10.1111/1556-4029.12987

Vredeveldt, A., Tredoux, C., Nortje, A., Kempen, K., Puljević, C, y Labuschagne, G. (2015). A field evaluation of the Eye-Closure Interview with witnesses of serious crimes. Law and Human Behavior, 39(2), 189-197. https://doi. org/10.1037/Ihb0000113

Wakefield, H. y Underwager, R. (1993). Misuse of psychological tests in forensic settings: Some horrible examples. American Journal of Forensic Psychology, 11(1), 55-75.

Willcock, E., Morgan, K. y Hayne, H. (2006). Body maps do not facilitate children's reports of touch. Applied Cognitive Psychology, 20(5), 607-615. https://doi. org/10.1002/acp.1212

Williamson, P., Weber, N. y Robertson, M. (2013). The effect of expertise on memory conformity: a test of informational influence. Behavioral Sciences and the Law, 31(5), 607-623. https://doi.org/10.1002/bsl.2094

Wolfman, M., Brown, D. y Jose, P. (2016). Talking past each other: Interviewer and child verbal exchanges in forensic interviews. Law and Human Behavior, 40(2), 107-117. https://doi.org/10.1037//hb0000171

Wright, D., Mathews, S. y Skagerberg, E. (2005). Social recognition memory: the effect of other people's responses for previously seen and unseen items. Journal of Experimental Psychology: Applied, 11(3), 200-209. https://doi. org/10.1037/1076-898X.11.3.200

Zaragoza, M., Payment, K., Ackil, J., Drivdahl, S. y Beck, M. (2001). Interviewing witnesses: forced confabulation and confirmatory feedback increase false memories. Psychologycal Science, 12(6), 473-477. https://doi. org/10.1111/1467-9280.00388

Zhu, B., Chen, C., Loftus, E., Lin, C. y Dong, Q. (2013). The relationship between DRM and misinformation false memories. Memory and Cognition, 41(6), 832-838. https://doi.org/10.3758/s13421-013-0300-2 